\title{
Development of Tests to Evaluate the Sensory Abilities of Children with Autism Spectrum Disorder using Touch and Force Sensors
}

\author{
M. Riederer, C. Schoenauer, H. Kaufmann \\ Institute of Software Technology and Interactive Systems \\ Vienna University of Technology, Vienna, Austria \\ \{riederer, schoenauer, kaufmann\}@ims.tuwien.ac.at
}

\author{
E. Soechting, C. Lamm \\ Department of Basic Psychological Research \\ University of Vienna, Vienna, Austria \\ \{a8102373, claus.lamm\}@unet.univie.ac.at
}

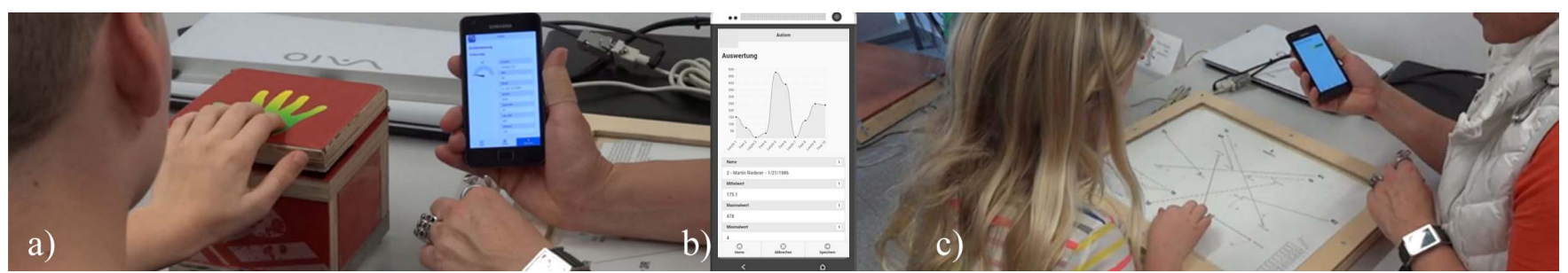

Fig. 1. The newly developed instruments in action: measuring proprioceptive functions in children

a) Grading of Force Test, b) Display of data on mobile device, c) Grading of Movement Test

\begin{abstract}
An emerging line of research that attempts to reveal underlying mechanisms of Autism Spectrum Disorder (ASD) studies differences in sensory processing in individuals with ASD. In this paper, we introduce new methods to measure proprioceptive functions of children with ASD. The instruments use a low-cost Arduino board and shield to acquire data from force and touch sensors. Data are transferred to mobile devices and analyzed with cross-platform application development tools. The instruments were pilot tested with typically developing children to test for functionality and usability of the instruments. They will be used in a larger study with children with ASD.
\end{abstract}

Keywords-autism spectrum disorder; proprioception; pervasive computing; mobile healthcare; wireless sensors

\section{INTRODUCTION}

Autism Spectrum Disorder (ASD) is a pervasive developmental disorder with an average prevalence of less than one percent [1]. It is characterized by impairments in social, communication and behavioral skills. Many countries prioritize autism research in order to better understand the causes and mechanisms of this disorder and to develop more specific and causal treatments compared to the dominating symptomatic approach of behavior modification. One line of research explores the sensory processing and sensorimotor differences in individuals with ASD [2, 3]. Differences in sensory processing and multisensory integration have been widely reported. Most of these studies used indirect measures such as parent questionnaires (e.g., the Sensory Profile [4]) for data collection. Novel test instruments that allow for direct measurement are warranted to substantiate these reports by "hard" objective data. Outcomes of this project could be translated into clinical applications (e.g., early detection of sensory processing disorders, focus of early intervention on underlying mechanisms)
One sensory system that has not been studied thoroughly is the proprioceptive sense. The proprioceptive sense carries information from receptors in muscles and joints. The primary source of proprioception is active muscle contraction. Proprioceptive information is used for regulation of tone and postural control, action model formation, motor planning and control, and behavioral organization [5].,,Given the importance of proprioception for even the most basic motor behaviors, directly examining proprioceptive processing in individuals with ASD is crucial for understanding the underlying causes of the social, communicative, and motor impairments. “ [6]

Our contributions to the field are:

- Design of tests and test instruments for objective assessment of proprioceptive functions.

- Implementation of a mobile low-cost system integrating these tests with sensors and mobile devices (see Fig. 1).

- Evaluation of the system's functionality und usability with typically developing subjects.

\section{MEDICAL BACKGROUND AND RELATED WORK}

Numerous studies have provided evidence that individuals with ASD process and use proprioceptive information differently (see [6], for an overview). Haswell et al. [7], found that children with ASD rely more on proprioceptive information to learn motor tasks. Only one study [6] investigated the type of sensory processing deficit that may impair effective use of proprioceptive information in autism. The findings of this study suggested that no primary proprioceptive deficits caused the motor impairment but maybe deficits at later processing states. This study used mostly passive tasks to measure proprioception. A new paradigm for examining proprioceptive processing at different processing stages that uses active muscle contraction may refine our understanding of the role of proprioception in autism. 
While there is solid evidence for deficits in participation, dyspraxia, and atypical use of proprioceptive information in children with ASD, the link between proprioceptive processing deficits, dyspraxia, and participation limitations has not yet been explored. For a future study that aims at revealing one of the deficient processes underlying praxis, we developed two objective measures of proprioceptive function, the Grading of Force (GoF) and the Grading of Movement (GoM) tests.

Computer technology plays an important role in the study of ASD for diagnostic and intervention purposes. One line of research relies on classical medical brain diagnosis tools, such as Magnetic Resonance Imaging (MRI) [8-10], while others assess motor functionality. Elnakib et al. [9] for example used shape analysis of 3D MRI images to detect atypical brain structures. Psychological approaches use eye and position tracking systems (e.g. Scassellati at Yale University) and social robots. Recent studies focusing on deviation of motor functions in children with ASD have shown promising results. Bugnariu et al. [11] analyzed lower limb function using treadmills and force sensors. Subjects had to balance, walk, and perform pointing and reaching tasks. A group at the University of Deusto [12] combined computer games and eye tracking technology to analyze interaction and motor skills of the upper extremities. Taffoni et al. [13] used Bluetooth connected sensors as a target to measure motor skills. Several studies use tangible objects of different shape with integrated sensors to examine how autistic individuals use haptic discrimination.

\section{DESIGN OF THE TESTS}

The two tests we developed are the Grading of Force test (GoF) and the Grading of Movement test (GoM). The GoF test is a measurement of how exactly the subject can grade his/her force. The subject's task is to exhibit pressure on a horizontal surface with the flat hand (distal portion of the 4 fingers). The GoM test, is a measurement of how precisely the subject can grade an arm movement without visual control. The task requires the subject to move his/her index finger from a starting point to a voluntary place on a horizontal surface. The GoM test is based on a paper-pencil test, the Kinesthesia test of the Sensory Integration and Praxis Tests [14]. The original test has been criticized for using passive movement. We eliminated this shortcoming in the design of our novel GoM test. Both tests are simple motor tasks that involve active muscle contraction and require minimal motor planning and are therefore considered to assess a relatively pure function of proprioception.

As described, one of the unique features of this system is that the stimulus is not presented by the examiner but created by the subject himself/herself. Compared to other systems, the advantage is that the devices do not need calibration providing a cost-efficient system with good usability in a clinical setting.

\section{A. Task Concept}

Test administration is similar for both the GoF and the GoM tests: after two practice items, 10 test items are administered, alternating left and right hand. 10 measurements are a typical number of probes to get a meaningful estimate of the subject's performance. Each item consists of a pair of two actions, stimulus and repetition. The first action of the subject (administering pressure respectively placing his/her index finger) serves as the stimulus that he/she attempts to repeat in his/her second action.
Since the subjects' vision is occluded, auditory feedback is given when the device has recorded the subject's action. The acoustic signal is different for action 1 (stimulus) and action 2 (repetition). The raw score for each item is the difference between action 1 and 2. The device records scores along with basic statistics such as max./min. values, average, median, and standard deviation.

\section{B. Grading of Force (GoF) Test}

The subject sits on a chair in front of a table with the device placed in front of him/her. The examiner explains the task verbally, demonstrates as necessary, and has the subject practice the two training items. The examiner places the subject's fingers on the device and encourages him/her to press down. During the training items, the subject receives visual feedback. He/she is encouraged to observe how a scale changes according to him/her changing the amount of force. Once the subject has understood the task, the 10 test items are administered, alternating left and right hand and without visual feedback.

The GoF device had to fulfill several requirements: Children are differing in age, gender and physical fitness, so the device needed to capture a wide range of force. After pilot testing, the maximum was set at 10 kilograms. Other requirements pertained to the accuracy of measurement and robustness of physical construction.

\section{Grading of Movement (GoM) Test}

The subject sits on a chair in front of a table. The touch screen is placed in front of the subject and the examiner guides the subject through the first training item by placing his/her finger on the starting point. The examiner asks the child to place his/her finger somewhere on the surface (action 1, stimulus), takes the subject's finger back to the starting point and has him/her repeat the movement, i.e. place the finger in the same place again. During the training, the examiner encourages the subject to visually monitor his/her action. After the training items the examiner places a cardboard shield on the device to occlude vision, and the 10 test items are administered, alternating hands. The verbal instructions include general descriptions of the type of movement (e.g. "Now place your finger far in my direction") to ensure that the subject varies his/her movements (long, short, ipsilateral, and crossing the midline to the contralateral side).

The technical requirements for the GoM device included the size of the touch pad, resolution and activation sensitivity. As these tests will be administered to autistic children, robustness of the devices was a basic requirement. Based on similar tests [14], we decided that the board size should be at least 18 inches in diagonal, to allow for whole arm movements and longer movement distances which are expected to result in greater deviations. Due to the physiological limits of children, for this test a capacitive touch screen with 20.65 inches in diagonal was chosen because of its higher sensitivity (see section IV.A).

\section{TECHNICAL IMPLEMENTATION}

The system was implemented as a hardware-software codesign that uses microcontrollers for sensor data acquisition and an application on a mobile device for analyzing and reporting tasks. The functional block diagram (see Fig. 2) 
shows the main components of the system. Each test has its own self-contained hardware, which measures sensor values and transmits data wirelessly to the main mobile device. On the mobile device data is processed, recorded, and analyzed.

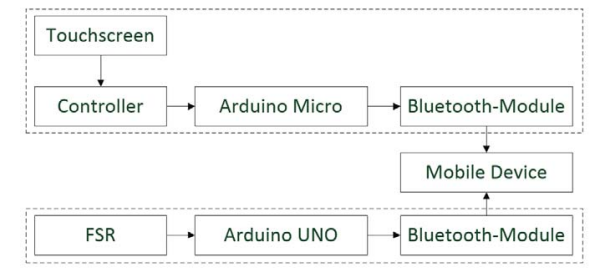

Fig. 2. Functional Block Diagram of the overall setup

\section{A. Hardware Setup}

Hardware design had to consider the medical requirements, ease of use (e.g. mobile devices, wireless connections), and low-cost setup. In both test setups, Arduino boards were used for data acquisition, in combination with add-on shields (see Fig. 4). We use Bluetooth shields for wireless transmission of the measured values, which communicate with the mobile device via Bluetooth Serial Protocol. Li-Po batteries allow autonomous operation.

In the GoF device, a Force Sensing Resistor (FSR) [15] is activated by a hinged wooden contactor. For better haptic feedback, we placed a piece of rubber between the sensor and the contactor (see Fig. 3). The FSR's active area has a side length of 1.7 inches and can process forces ranging from 0.1 to $10 \mathrm{~kg}$. A simple resistive voltage divider measures the sensor value. The output voltage of the divider is digitized by an Arduino UNO board and sent to the mobile device in a continuous stream. The system is able to detect touch-down and lift-off events on the device and only sends data if force is applied.

The GoM device integrates a transparent capacitive touch screen and the dedicated controller in a robust wooden frame. The comprised $3 \mathrm{M}^{\mathrm{TM}}$ MicroTouch ${ }^{\mathrm{TM}}$ capacitive touch system has a diagonal size of 20.65 inch and is very robust with regard to force added to its surface. Positional data from the touch screen is gathered via a serial interface controller $3 \mathrm{M}^{\mathrm{TM}}$ UC7720SC, which is connected to an Arduino Micro board by a RS232 connection (see Fig. 4). Because the Arduino system only handles TTL-level signals, we installed a Serial-to-TTL converter. A Bluetooth module transmits the processed data to the mobile device.
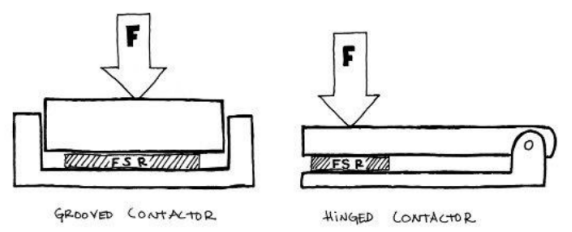

Fig. 3. FSR attachment options for load transmission - Our implementation uses a hinged contactor [16]

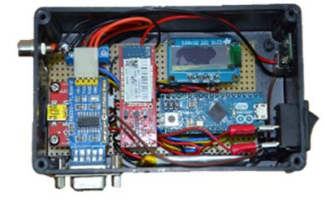

Fig. 4. Data acquisition unit of the GoM device

\section{B. Software Implementation}

The system's software implementation can be divided in two main levels: the implementation of data acquisition on the Arduino boards, and the data analysis and reporting application on the mobile device.

The main aspect in the Arduino implementation is the conversion of the measured sensor values to usable values for the reporting application. As the FSR sensors resistance is not linear to the applied force, a conversion algorithm had to be implemented. This algorithm maps the digitized values to the reference voltage range and calculates the conductance of the FSR. Furthermore, the values from the touch screen controller with a default resolution of $1024 \times 1024$ are mapped to a suitable range $(1920 x 1080)$ to fit the touch screen's 16:9 form factor.

Cross-Platform development and mobile applications in healthcare are getting more and more popular and allow mobile data acquisition for medical professionals on a variety of devices [17]. We used HTML5 and JavaScript for realizing our mobile application. For an easier and faster development process jQuery Mobile has been utilized. Access to hardwarelevel features is implemented using the Adobe $\AA$ PhoneGap Framework [18].

The main features of our mobile application are:

- Management of patient data

- Connection to Bluetooth devices

- Guided sequences for GoF and GoM tests

- Recording of evaluation sessions

- Calculation of basic statistical values and visualization

- Exporting of saved sessions

\section{Test Implementation}

The application supports and guides the therapist and patient throughout the whole test sequence including training and measurement as described in section III. After completion of the tasks, the software analyses the whole set of data and computes the following data: arithmetic mean value, median, maximum, minimum, variance and standard deviation. The data is saved on the local memory of the mobile device as a CSV-file for further analysis. First tests have shown that feedback is very important to notify about task and context changes. Therefore, our application generates different audio signals as described in subsection III.A. Visualization of a gauge meter in the GoF test gives feedback to the therapist (and the patient in the training phase) by showing the applied force. In the GoM test every touch is visualized in a downscaled version on the display of the mobile device, so the therapist gets feedback about the test's progress. 


\section{Evaluation}

We assessed the functionality and workflow of our setup in a pilot test with typically developing children and absolute measurements. First we evaluated the precision of the GoF device by applying precisely predetermined weights instead of manual pressure. The test consisted of 4 runs with 10 different weights between $0.25 \mathrm{~kg}$ and $8 \mathrm{~kg}$. As shown in Fig. 5 the measured sensor values increase almost linearly with the added weight, which facilitates relating measurements to weight. The average deviation from the mean values over all weights is $1.84 \%$, demonstrating sufficient repeatability and accuracy.

The GoM device was evaluated by two experiments: First a child touched a single point at least 4 times, then it touched the two end points of a line. In the single touch test we conducted 8 runs at 3 different point positions, where we measured an average deviation of $0.37 \%(3 \mathrm{~mm})$ from the mean value. For the second test we drew lines of 50 and $100 \mathrm{~mm}$ on a sheet of paper and placed it under the touch screen. The position of the sheet was varied for 3 times in 72 runs. Overall we captured 144 points and evaluation of the data showed a deviation from the predefined line length of $4.69 \%$ (3.5mm). Therefore, accuracy and repeatability can be considered sufficient and are comparable with paper-pencil tests such as [14].

Furthermore, the instruments and workflow was pilot tested with three typically developing children ( 1 female, age 8 years, 2 male, age 10 and 11 years) in cooperation with a psychologist (examiner). The subjects completed 10 trials on the GoF-Test following 10 trials on the GoM-Test. Data gained from these trials are shown in Table I. Examiner and subjects found the administration of the two tests utilizing the instruments feasible. Further studies with larger groups of typical and autistic children are needed to establish their discriminative properties and clinical utility.

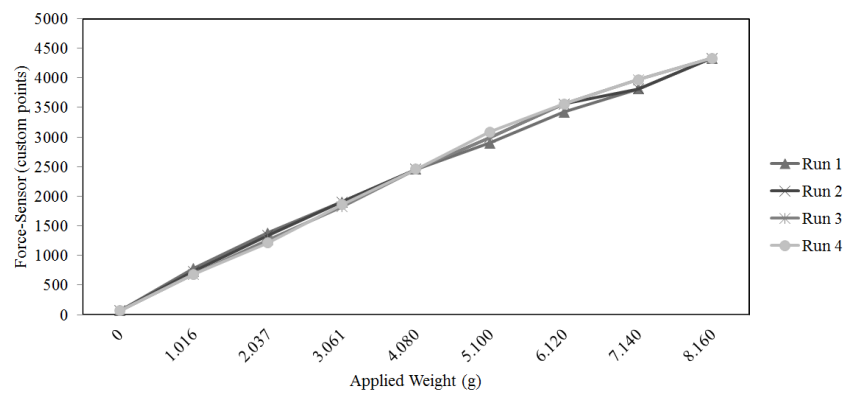

Fig. 5. Chart showing the relationship between added weight and force sensor output values

\section{CONCLUSION}

In this paper we introduced the design and implementation of an instrument that evaluates proprioceptive functions, i.e. grading of force and movement without visual control. We expect that these measurements will discriminate between children with ASD and typical children. The instrument is based on a low-cost Arduino board and wireless Bluetooth transfer of data to a mobile device where a mobile application .reports and calculates statistical data. The system was evaluated for functionality and accuracy and was pilot tested with typical children. If the instrument proves to provide meaningful data that discriminate between children with ASD and typical children, it shall be made available for clinical use.
TABLE I

EVALUATION RESULTS, RANGE OF RAW SCORES FOR GOF $0-$ 5000, RANGE OF RAW SCORES FOR GOM 0-26

\begin{tabular}{|c|r|r|r|r|}
\hline Person & \multicolumn{2}{|c|}{ GoF } & \multicolumn{2}{c|}{ GoM } \\
\hline & Avg. & \multicolumn{1}{c|}{ Min. } & \multicolumn{1}{c|}{ Avg. } & \multicolumn{1}{c|}{ Min. } \\
\hline Girl & 341 & 0 & 20 & 7 \\
\hline Boy 1 & 253 & 40 & 7 & 0 \\
\hline Boy 2 & 303 & 75 & 26 & 1 \\
\hline
\end{tabular}

\section{REFERENCES}

[1] J. Baio, "Prevalence of Autism Spectrum Disorders: Autism and Developmental Disabilities Monitoring " Centers for Disease Control and Prevention, Network, 14 Sites, United States 1546-0738, 2012.

[2] A. M. Donnellan, D. A. Hill, and M. R. Leary, "Rethinking autism: implications of sensory and movement differences for understanding and support," Frontiers in Integrative Neuroscience, vol. 6, 2013-January-28 2013

[3] E. J. Marco, L. B. N. Hinkley, S. S. Hill, and S. S. Nagarajan, "Sensory Processing in Autism: A Review of Neurophysiologic Findings," Pediatr Res, vol. 69, pp. 48R-54R, 2011.

[4] W. Dunn, "The Sensory Profile manual," San Antonio1999.

[5] U. Proske, "What is the role of muscle receptors in proprioception?," Muscle \& nerve, vol. 31, pp. 780-787, 2005.

[6] C. Fuentes, S. Mostofsky, and A. Bastian, "No Proprioceptive Deficits in Autism Despite Movement-Related Sensory and Execution Impairments," Journal of Autism and Developmental Disorders, vol. 41, pp. 1352-1361, 2011/10/01 2011.

[7] C. C. Haswell, J. Izawa, L. R. Dowell, S. H. Mostofsky, and R. Shadmehr, "Representation of internal models of action in the autistic brain," Nat Neurosci, vol. 12, pp. 970-972, 2009.

[8] S. Vigneshwaran, B. S. Mahanand, S. Suresh, and R. Savitha, "Autism spectrum disorder detection using projection based learning metacognitive RBF network," in The International Joint Conference on Neural Networks (IJCNN), 2013, pp. 1-8.

[9] A. Elnakib, M. F. Casanova, G. Gimel'farb, A. E. Switala, and A. ElBaz, "Autism diagnostics by centerline-based shape analysis of the Corpus Callosum," in IEEE International Symposium on Biomedical Imaging: From Nano to Macro, 2011, pp. 1843-1846.

[10] Y. Takarae, B. Luna, N. J. Minshew, and J. A. Sweeney, "Visual Motion Processing and Visual Sensorimotor Control in Autism," Journal of the International Neuropsychological Society, vol. 20, pp. 113-122, 2014.

[11] N. Bugnariu, C. Garver, C. de Weerd, E. van Loon, C. Young, K. Rockenbach, R. Longnecker, and R. M. Patterson, "Motor function in children with Autism spectrum disorders," in International Conference on Virtual Rehabilitation (ICVR), 2013, pp. 51-56.

[12] N. Aresti Bartolome, A. Mendez Zorrilla, and B. Garcia Zapirain, "Autism Spectrum Disorder children interaction skills measurement using computer games," in 18th International Conference on Computer Games: AI, Animation, Mobile, Interactive Multimedia, Educational \& Serious Games (CGAMES), 2013, pp. 207-211.

[13] F. Taffoni, V. Focaroli, D. Formica, E. Gugliemelli, F. Keller, and J. M. Iverson, "Sensor-based technology in the study of motor skills in infants at risk for ASD," in 4th IEEE RAS \& EMBS International Conference on Biomedical Robotics and Biomechatronics (BioRob), 2012, pp. 18791883.

[14] A. J. Ayres, "The Sensory Integration and Praxis Tests," Los Angeles, CA1989.

[15] S. I. Yaniger, "Force Sensing Resistors: A Review Of The Technology," in Electro International, 1991, pp. 666-668.

[16] O. M. Labs. (2014, 22.02.2014). Force Sensitive Resistors (FSRs). Available: http://www.openmusiclabs.com/learning/sensors/fsr/

[17] A. Ellertson, "Work in progress: Using smart mobile tools to enhance autism therapy for children," in Frontiers in Education Conference (FIE), 2012, pp. 1-2.

[18] R. Mahesh Babu, M. B. Kumar, R. Manoharan, M. Somasundaram, and S. P. Karthikeyan, "Portability of mobile applications using PhoneGap: A case study," in International Conference on Software Engineering and Mobile Application Modelling and Development (ICSEMA), 2012, pp. 16. 